\title{
Benzotriazole ultraviolet stabilizers alter the expression of the thyroid hormone pathway in zebrafish (Danio rerio) embryos
}

\author{
Xuefang Liang ${ }^{\text {a, * }}$, Jiajia Li ${ }^{\text {a }}$, Christopher J. Martyniuk ${ }^{\mathrm{d}}$, Juan Wang a , Yufeng Mao a , \\ Huan Lu ${ }^{a}$, Jinmiao Zha ${ }^{b, c}$ \\ a School of Ecology and Environment, Inner Mongolia University, Hohhot, 010021, China \\ ${ }^{\mathrm{b}}$ Key Laboratory of Drinking Water Science and Technology, Research Center for Eco-Environmental Sciences, Chinese Academy of Sciences, Beijing, 100085, \\ China \\ ${ }^{\mathrm{c}}$ Beijing Key Laboratory of Industrial Wastewater Treatment and Reuse, Research Center for Eco-Environmental Sciences, Chinese Academy of Sciences, \\ Beijing, 100085, China \\ ${ }^{\mathrm{d}}$ Department of Physiological Sciences and Center for Environmental and Human Toxicology, College of Veterinary Medicine, UF Genetics Institute, \\ University of Florida, Gainesville, FL, 32611, USA
}

\section{H I G H L I G H T S}

- BUVSs decreased heart rate of zebrafish in all treatments by $6.9 \%-21.4 \%$.

- BUVSs affected transcriptional levels of HPT axis-related genes.

- Disruption of thyroid-related transcripts was dependent on the structure of BUVSs.

- Gene networks and pathways were predicted using IMP.

\section{A R T I C L E I N F O}

\section{Article history:}

Received 19 March 2017

Received in revised form

30 April 2017

Accepted 2 May 2017

Available online 3 May 2017

Handling Editor: David Volz

\section{Keywords:}

Benzotriazole ultraviolet stabilizers

Thyroid hormone receptor

HPT axis-related genes

Zebrafish

Toxicological pathways

\begin{abstract}
A B S T R A C T
Benzotriazole ultraviolet stabilizers (BUVSs) are widely used in industrial products as well as personalhygiene products to protect the material or skin from harmful UV-radiation. Due to their persistence and bioaccumulation, BUVSs have been ubiquitously detected in aquatic environments. Although the toxicological effects of BUVSs in aquatic organisms have been previously examined, the effects of BUVSs on the thyroid system have not been adequately addressed. In this study, we assessed putative thyroid disrupting effects of BUVSs (UV-234, UV-326, UV-329 and UV-P) in zebrafish embryos at 1, 10 and $100 \mu \mathrm{g} / \mathrm{L}$ for $96 \mathrm{~h}$. The heart rate was assessed in zebrafish and was observed to be decreased by $6.9 \%$ $-21.4 \%$ in exposure of tested BUVSs. We also observed that the transcript levels of HPT axis-related genes were affected by the 4 BUVSs tested in different ways. Specifically, mRNA levels of thyroid hormone receptors (thraa and thrb) in zebrafish embryos were differentially expressed and the direction of change in these transcripts was isoform and BUVSs dependent. Pathway analysis of the targeted genes measured indicated that cellular processes putatively affected by BUVSs included response to organic substance, regulation of transcription from RNA polymerase II promoter, intracellular receptor signaling pathway, and hypothyroidism. Upon expansion of the network, novel genes involved in this predicted gene network may provide insight into the mechanisms of thyroid disrupting mechanisms of BUVSs. Taken together, our results indicate that BUVSs can potentially impact the thyroid system, and that this is dependent upon the type or structure of BUVSs.
\end{abstract}

(C) 2017 Elsevier Ltd. All rights reserved.
* Corresponding author. School of Ecology and Environment, Inner Mongolia University, 235 West College Road, Hohhot, Inner Mongolia, 010021, China.

E-mail address: liangxf@imu.edu.cn (X. Liang).

\section{Introduction}

Benzotriazole ultraviolet stabilizers (BUVSs) have a structural feature of a benzotriazole moiety attached with a phenolic group and are often referred to as Tinuvin (Kawamura et al., 2003). These compounds absorb the full spectrum of UV-light (UV-A and UV-B) 
from 280 to $400 \mathrm{~nm}$, and are used in manufacturing to protect materials against harmful UV-radiation and structural damage (Nakata et al., 2009). BUVSs have been applied in textiles, plastics, building materials, automobile components, paint, and sports equipment to prevent yellowing and degradation (Nakata et al., 2009). In addition, they are also used in personal-hygiene products, including body lotions, creams, shampoos, fragrances, and sunscreens (Kim et al., 2011a; Nagayoshi et al., 2015). Due to their high production volume and wide application, BUVSs have been frequently detected in various environmental matrices such as municipal waste water, sewage sludge, sediment, soil, and indoor dust (Lai et al., 2014; Ramos et al., 2016; Wang et al., 2013a). Most BUVSs are detected in aquatic environments at mean concentrations ranging from 2.3 to $544.9 \mathrm{ng} / \mathrm{L}$ (Carpinteiro et al., 2010, 2012; García-Guerra et al., 2016; Kameda et al., 2011; Liu et al., 2011; Montesdeoca-Esponda et al., 2012; Song et al., 2014). Among these BUVSs, UV-326, UV-327, and UV-328 appear to be the most frequently detected compounds in environmental samples (Cantwell et al., 2015; Kameda et al., 2011; Langford et al., 2015; Liu et al., 2011; Nakata et al., 2009; Ruan et al., 2012; Song et al., 2014; Wick et al., 2016; Zhang et al., 2011). UV-234 and UV-329 were recently reported to be found in sewage sludge in China with an average of 116 and $66.8 \mathrm{ng} / \mathrm{g} \mathrm{dw}$ (dry weight) respectively (Ruan et al., 2012). Moreover, UV-234 concentration in sediments from Japanese rivers has been detected upwards of $363 \mathrm{ng} / \mathrm{g} \mathrm{dw}$ (Kameda et al., 2011), whereas UV-329 was detected up to $2075.5 \mathrm{ng} / \mathrm{g} \mathrm{dw}$ in sewage sludge in Norway (Langford et al., 2015). Thus, high concentration and persistence of these compounds in sediment and sewage sludge suggest that these matrices can be a significant source of BUVSs contamination for aquatic ecosystems.

Due to their highly hydrophobic properties ( $\log$ Kow $>4.31$ ), some BUVSs are highly persistent and show the potential to be accumulated in aquatic organisms and human. BUVSs have been widely detected in fish, aquatic birds, and macroinvertebrates from Japanese coastal waters, Manila Bay, Philippines, as well as in other water systems (Kim et al., 2011b, 2011c; Lu et al., 2016; Nakata et al., 2010, 2012; Wick et al., 2016). Additionally, BUVSs have been reported in human breast milk (Lee et al., 2015), adipose tissue (Wang et al., 2015), as well as urine (Asimakopoulos et al., 2013a, 2013b), suggesting that there is internal exposure to these compounds and that they are being actively metabolized and excreted. The persistence and bioaccumulation of BUVSs raise concern about their potential adverse effects to human.

There has been limited reports of the toxicological impacts of BUVSs; these compounds appear to have low acute toxicity in Daphnia $\left(\right.$ LC $_{50}>10 \mathrm{mg} / \mathrm{L}$ ) (Kim et al., 2011a). Recent studies in mammals and fish have reported on their endocrine disrupting effects (Fent et al., 2014; Kawamura et al., 2003; Morohoshi et al., 2005; Nagayoshi et al., 2015; Zhuang et al., 2017). UV-P, UV-9, UV-326, and UV-090 show significant ligand activity against human aryl hydrocarbon receptor (AhR) (Nagayoshi et al., 2015). In addition, biotransformation of BUVSs has been shown to influence their anti-androgenic activities, thus bioactivation/biotransformation appear to be important mechanisms that determine their toxicity and endocrine disrupting actions (Zhuang et al., 2017). These studies suggest that the various BUVSs can exert structureassociated effects in human and aquatic organisms.

In terms of their toxicity, UV-P and UV-326 are involved in the activation of the AHR-pathway in zebrafish embryos (Fent et al., 2014). Additionally, mRNA level of thyroid hormone receptor $\alpha$ in zebrafish embryos was observed to be significantly decreased following exposure to $690 \mu \mathrm{g} / \mathrm{L}$ UV-P (Fent et al., 2014), whereas, none of the tested BUVSs showed ligand activity against the human thyroid hormone receptors (Nagayoshi et al., 2015). Thyroid hormones (THs) play fundamental roles in regulating development, differentiation, and metabolism of all vertebrates (Power et al., 2001). THs act by binding to specific nuclear receptors and play important roles for embryonic to larval transitory phase in fish (Liu and Chan, 2002). The hypothalamic-pituitary-thyroid (HPT) axis plays a key role in developmental programming and is responsible for maintaining homeostasis of THs (Blanton and Specker, 2007). The HPT is also an important target of endocrine-disrupting chemicals (Nichols et al., 2011). With the introduction of molecular biology techniques, HPT axis-related genes have been used as sensitive endpoints to assess chemicals that can have significant detrimental effects on the HPT axis (Porazzi et al., 2009). However, currently, there has been very limited work conducted to investigate the effects of BUVSs in the HPT axis of fish, and to investigate whether the impacts of structurally similar analogs of BUVSs are consistent. As a widely used vertebrate model organism, zebrafish and its embryos have been applied in assessment of thyroiddisrupting chemicals (Raldúa et al., 2012). In the present study, we examined the effects of four BUVSs (UV-234, UV-326, UV-329 and UV-P) on zebrafish embryos. As mentioned above, these compounds were most frequently detected or exist at relative high levels in environment compared to other BUVSs. The developmental toxicity and gene transcription responses in the HPT axis were assessed. Furthermore, cluster and pathway analysis was performed using expression patterns in order to synthesize data and to develop improved understanding for endocrine disruption of the thyroid hormone axis by BUVSs.

\section{Materials and methods}

\subsection{Chemicals}

UV-stabilizers UV-P (CAS no. 2440-22-4, purity >99\%), UV-234 (CAS no. 70321-86-7, purity >99\%), UV-326 (CAS no. 3896-11-5, purity $>99 \%$ ), UV-329 (CAS no. 3147-75-9, purity $>98 \%$ ) were purchased from J\&K Chemical Ltd. (USA). All structures and physiochemical properties are given in Table 1. Stock solution of BUVSs were prepared by dilution in HPLC-grade acetone and then mixed with distilled-deionized water. A vehicle treatment containing a combination of acetone served as a control. The ratio of vehicle to water was $1: 100,000(\mathrm{vol} / \mathrm{vol})$.

\subsection{Experimental design}

Adult zebrafish (Wild type $A B$ ) were raised in a flow-through system with dechlorinated tap water $(\mathrm{pH}$ 7.2-7.6; hardness $\left.44.0-61.0 \mathrm{mg} \mathrm{CaCO}_{3} / \mathrm{L}\right)$ at a constant temperature $\left(26 \pm 1^{\circ} \mathrm{C}\right)$ with a photoperiod of $14: 10 \mathrm{~h}$ (light: dark). Zebrafish were randomly selected from a breeding stock and placed in a shallow water breeding tank the night before embryo collection. The ratio used for breeding was three males to one female per tank and there were four tanks used to generate fertilized embryos. The facility lights were turned on at 8:00 a.m. and spawning occurred. Embryos were pooled in 2 petri dishes, and washed twice using dechlorinated tap water. Using a light microscope, unfertilized eggs were identified and removed. The fertilized eggs ( $300-400$ per spawning) in the same stage (shield stage, 6-8 h post fertilization, hpf) were selected and used in the following exposure experiments. Embryos were staged using the criteria of Kimmel et al. (1995).

Twenty embryos were transferred to each well of a 6-well culture plate filled with $8 \mathrm{~mL}$ exposure solution. Three well replicates were performed for each treatment. For each chemical, 3 doses were investigated $(1,10,100 \mu \mathrm{g} / \mathrm{L})$. The test concentrations of chemicals were selected based upon data presented in Fent et al. (2014). Embryos were maintained in an incubator at $26 \pm 1{ }^{\circ} \mathrm{C}$ and exposed to the same light to dark schedule as above. For the 
Table 1

List of BUVSs, CAS Registry Number, IUPAC name, chemical structures, log Kow (octanol/water partition coefficient) values and molecular weight.

\begin{tabular}{|c|c|c|c|c|c|}
\hline Compounds & CAS\# & IUPAC name & Chemical structures & $\log$ Kow & Molecular weight \\
\hline UV-P & $2440-22-4$ & 2-(benzotriazol-2-yl)-4-methylphenol & & 4.31 & 225.25 \\
\hline UV-234 & $70321-86-7$ & $\begin{array}{l}\text { 2-(benzotriazol-2-yl)-4,6-bis } \\
\text { (2-phenylpropan-2-yl)phenol }\end{array}$ & & 7.67 & 447.57 \\
\hline UV-326 & $3896-11-5$ & 2-tert-butyl-6-(5-chlorobenzotriazol-2-yl)-4-methylphenol & & 5.55 & 315.81 \\
\hline UV-329 & $3147-75-9$ & 2-(benzotriazol-2-yl)-4-(2,4,4-trimethylpentan-2-yl)phenol & & 6.21 & 323.43 \\
\hline
\end{tabular}

duration of the experiment, embryos were not disturbed, and this constituted a static waterborne exposure over 96 h with $90 \%$ water renewal with the chemical each day. Larvae in each group were collected after 96-h exposure. Five larval fish were randomly collected from the same well and pooled in one centrifuge tube as one biological replicate ( $n=3 /$ treatment) for gene expression analysis. Once pooled, samples were flash-frozen using liquid nitrogen and stored at $-80^{\circ} \mathrm{C}$ until subsequent RNA extraction.

\subsection{Embryo development and heart beat}

To assess the developmental toxicity of BUVSs on zebrafish embryos, mortality, malformations, and heartbeat rate (72hpf) were recorded. During exposure, developmental parameters were monitored and documented daily between 24 and $96 \mathrm{hpf}$ according to Reimers et al. (2004). All embryos were staged as previously described (Kimmel et al., 1995). Heartbeat rates were evaluated in 6 embryos per well over a $20 \mathrm{~s}$ interval at $72 \mathrm{hpf}$. All the observations above were conducted using an Olympus SZX16 microscope (Olympus Corp., Tokyo, Japan) equipped with an NIKON D5100 digital camera.

\subsection{RNA extraction and CDNA synthesis}

Total RNA was isolated from zebrafish embryos using $1 \mathrm{~mL}$ TRIzol reagent (Life Technology) as per manufacturer's protocol. The integrity of total RNA was visually examined using $1.2 \%$ agarose gel electrophoresis $(0.5 \times$ TBE buffer, $150 \mathrm{~V}, 15 \mathrm{~min})$. The concentration of RNA was determined using a Multiskan ${ }^{\mathrm{TM}} \mathrm{GO}$ microplate spectrophotometer (Thermo Scientific, USA) and the $\mathrm{OD}_{260} / \mathrm{OD}_{280}$ ratio was examined to confirm sample purity. The RNA samples were dissolved in ribonuclease-free water and stored at $-80^{\circ} \mathrm{C}$.

The cDNA was synthesized with $2 \mu \mathrm{g}$ total DNAse treated RNA from each sample according to the manufacturer's instructions
(Promega). Samples were placed in a Veriti Thermal Cycler (Applied Biosystems, USA). The cDNA was generated using the following steps: $25^{\circ} \mathrm{C}$ for $5 \mathrm{~min}, 42^{\circ} \mathrm{C}$ for $30 \mathrm{~min}, 70^{\circ} \mathrm{C}$ for $15 \mathrm{~min}$, and a final cycle of $4{ }^{\circ} \mathrm{C}$ for $5 \mathrm{~min}$.

\subsection{Relative quantification by real-time PCR}

Real-time PCR was performed using the CFX96 ${ }^{\mathrm{TM}}$ Real-Time PCR Detection System (BioRad) in a total volume of $20 \mu \mathrm{l}$, consisting of the GoTaq $^{\circledR}$ qPCR Master Mix (Promega, USA), $200 \mathrm{nM}$ forward primer and $200 \mathrm{nM}$ reverse primer. The cycling conditions used were: an initial denaturation step of $95{ }^{\circ} \mathrm{C}$ for $10 \mathrm{~min}$; 40 cycles of $95^{\circ} \mathrm{C}$ for $30 \mathrm{~s}, 57^{\circ} \mathrm{C}$ for $40 \mathrm{~s}$, and $72{ }^{\circ} \mathrm{C}$ for $30 \mathrm{~s}$; and the last cycle of $95{ }^{\circ} \mathrm{C}$ for $30 \mathrm{~s}, 57{ }^{\circ} \mathrm{C}$ for $30 \mathrm{~s}$, and $72{ }^{\circ} \mathrm{C}$ for $60 \mathrm{~s}$ to generate the dissociation curve. An exclusive peak was observed for each amplification, which indicated that there was no amplification of untargeted genes. The control, which contained all of the reaction components except for the template, was included in all experiments.

Five reference genes (18s, $\beta$-actin, ef $1 \alpha$, rpl8 and rpl13a) were assessed for stability and appropriateness for normalization. The geNorm V3.5 was adopted to identify the most stable reference genes. Genes with the lowest M-values were selected as candidate reference genes. The housekeeping gene ribosomal protein L13 $\alpha$ (rpl13a) was determined to be the most stable reference gene to normalize all target genes (data not shown). For all cDNA samples, 3 biological replicates were assessed for each concentration. The mean values were used for calculations of relative mRNA levels using the delta-delta Ct method (Livak and Schmittgen, 2001). Supplemental Table S1 lists the primer pairs used for real-time PCR.

\subsection{Cluster analysis}

A cluster analysis (JMP Genomics v 5.0, SAS) was conducted in 
order to determine how transcriptional responses in the zebrafish embryos responded overall to the different concentrations of 4 BUVSs. Prior to hierarchical clustering using the Fast Ward algorithm, each column of expression data (relative fold change for each gene at each dose) was scaled and centered to produce Z-scores. This approach subtracts the mean for each column and then divides by the column standard deviation in order to standardize all data prior to clustering. In addition, each row was centered to a mean of zero (0) and scaled to a variance of one (1) prior to clustering. We also used the normalized expression of each transcript to determine whether there were expression patterns unique to the different UV-stabilizers.

\subsection{Biological process enrichment analysis}

To identify novel gene participants and pathways potentially associated with the transcripts investigated, a gene set including all target genes except for tpo and trhr (which were not mapped to the program) were submitted to IMP (Integrative Multi-species Prediction, http://imp.princeton.edu/). Additionally, prediction of gene functions and networks was performed using IMP. For functionally related gene network prediction, minimum relationship confidence was set to 0.1, and the maximum number of genes was 25. Biological networks were predicted with a confidence cutoff of 0.5 .

\subsection{Statistical analysis}

All quantitative data are expressed as mean \pm S.E. Statistical analysis of variance (ANOVA) was performed using SPSS (version 17.0). The normality of data was assessed using the KolmogorovSmirnov test and logarithmic transformation was performed prior to ANOVA to ensure data conformed to assumptions. The Levene's test of homogeneity of variance was used to test for adherence to ANOVA assumptions. For heartbeat rates, Tukey's test was used to compare data between treatments. For qPCR, a Dunnett's post-hoc test was used to compare each treatment with the control. A probability of $\mathrm{p}<0.05$ was considered to be statistically significant.

\section{Results}

\subsection{Effects of BUVSs on development and heart rates in zebrafish embryos}

During the $96 \mathrm{~h}$ exposure, mortality was monitored daily and recorded. At the end of the 96-h exposure period, no significant mortality was observed in any treatment compared to control (percentage of mortality $<5 \%$, data not shown). Embryonic malformations were observed following the 24- and 96-h BUVSs exposures. Endpoints monitored included development delay, axial edema, axial malformations (crooked/clubbed), otolith defects, pericardial edema, yolk sac edema, and disturbance of pigmentation. Deformities were observed in the $10 \mu \mathrm{g} / \mathrm{L} \mathrm{UV}-234$ and $10 \mu \mathrm{g} / \mathrm{L}$ UV-329 groups, but the prevalence of the deformities was not statistically different from the controls (percentage of malformation $<5 \%$, Fig S1).

Significant effects on heartbeat rate were detected in zebrafish embryos exposed to all 4 BUVSs (Fig. 1). In the UV-326 and UV-329 treatment groups, heartbeat rates were significantly decreased by $13.6 \%-21.4 \%$ at all concentrations investigated compared to the control group (Fig. 1a and c). After UV-234 exposure, heartbeat rates of zebrafish were significantly inhibited by $13.1 \%$ and $16.3 \%$ in 10 and $100 \mu \mathrm{g} / \mathrm{L}$ groups, respectively (Fig. 1b). Heartbeat rates were significantly decreased by $11 \%$ in $100 \mu \mathrm{g} / \mathrm{L}$ UV-P; however, there were no remarkable differences observed in the lower doses (Fig. 1d).

\subsection{Transcriptional levels of the HPT axis-related genes}

The expression levels of corticotropin releasing hormone (crh), iodothyronine deiodinases (dio1 and dio2), thyroid transcription factor-1 ( $n k \times 2.1$ ), solute carrier family 5 (sodium/iodide cotransporter), member 5 (slc5a5), thyroglobulin ( $\operatorname{tg}$ ), thyroid hormone receptors (thraa and thrb), thyroid peroxidase (tpo), thyrotropinreleasing hormone (trh), thyrotropin-releasing hormone receptor (trhr), thyroid stimulating hormone $\beta$ (tshb), thyroid stimulating hormone receptor (tshr), transthyretin (ttr), and uridine diphosphate glucuronosyltransferase (ugt1ab) were determined (Table S2, and Fig. 2). The cluster analysis showed that the response of transcripts related to the HPT axis in fish exposed to UV-234 and UV329 was most similar among chemicals while the response pattern of UV-326 was most distinct (Fig. 3).

For UV-326, the transcript levels of all genes tested, except for $t t r$, were generally increased in the embryos in all exposure groups (Table S2, and Fig. 2). The expression level of ttr was significantly decreased by 0.33 - fold with $100 \mu \mathrm{g} / \mathrm{L} \mathrm{UV}-326$ treatment ( $<<0.05$ ), while this transcript was up-regulated by 2.7 - and 2.8 - fold after exposure to $10 \mu \mathrm{g} / \mathrm{L}$ and $100 \mu \mathrm{g} / \mathrm{L}$ of UV-329 (Table S2). In addition, thraa ( 3.6-3.7- fold, p < 0.05), thrb (2.7- fold, $\mathrm{p}<0.05$ ), and trhr (4.4-4.6-fold, $\mathrm{p}<0.05$ ) were significantly increased in one or more concentration of UV-326 (Fig. 4). However, in the UV-329 treatment, thraa (2.2- fold, p < 0.05) and thrb (2.4- fold, p < 0.05) were significantly up-regulated in fish, but only at the highest dose examined while trhr was markedly down-regulated across all concentrations by $52 \%-60 \%$ ( $p<0.05$ ) (Fig. 4). For UV-234, the mRNA levels of thrb were increased by $2.2-$ and $2.4-$ fold in $10 \mu \mathrm{g} / \mathrm{L}$ and $100 \mu \mathrm{g} / \mathrm{L}$ groups, respectively; while no changes were observed for thraa (0.5-1.3- fold, $\mathrm{p}>0.05)$ and trhr (0.7-1.1- fold, $\mathrm{p}>0.05)$ (Table S2, and Fig. 4). After exposure of UV-P, expression levels of thrb (0.5- fold, $\mathrm{p}<0.05)$ and trhr $(0.3$-fold, $\mathrm{p}<0.05)$ were significantly reduced in $10 \mu \mathrm{g} / \mathrm{L}$ treatment, while no alteration was shown in the mRNA steady state levels of thraa $(0.8-1.2$ - fold, $\mathrm{p}>0.05)$ (Fig. 4).

Notably, the transcription levels of tshb $(0.45$ - fold, $\mathrm{p}<0.05)$ and slc5a5 (0.2-0.4- fold, $\mathrm{p}<0.05)$ in UV-234 treatments were significantly down-regulated in $1 \mu \mathrm{g} / \mathrm{L}$ and all three doses, respectively. However, no significant differences were observed in the other 3 BUVSs treatment groups (Table S2, and Fig. 2).

\subsection{Prediction of gene networks of substances}

In order to predict biological processes related to the transcripts affected by BUVSs, IMP was used to analyze the HPT axis-related genes examined here. Novel genes found in the gene networks that interact with the gene set included arginine vasopressin-like (avpl), runt-related transcription factor $2 \mathrm{~b}($ runx $2 b)$, urotensin 1 (uts1), dopamine receptor D2b ( $d r d 2 b)$, aristaless related homeobox (arx), among others (Fig. 5a and Table S3). High confidence scores between these genes to our targeted gene list indicate that they are highly associated with the same biological processes. For example, confidence scores between runx $2 b$ and tshb, uts 1 and $c r h$, and $n k x 2.1 a$ and arx were $0.82,0.78,0.91$, respectively (Table S4). In addition, estrogen receptor 1 (esr1), corticotropin releasing hormone receptor 1 (crhr1), and UDP glucuronosyltransferase 1 family, polypeptide A3 (ugt1a3) showed a high possibility $(0.88,0.63$, and 0.52 , respectively) of interacting with the target genes (Table S5). Additionally, pathway prediction revealed that these genes are likely involved in hypothyroidism, response to immobilization stress, regulation of female receptivity, regulation of cardiac chamber formation and morphogenesis, and cardiac muscle cell proliferation (Table S6). The main biological themes that the targeted transcripts shared among each other included response to 
a)

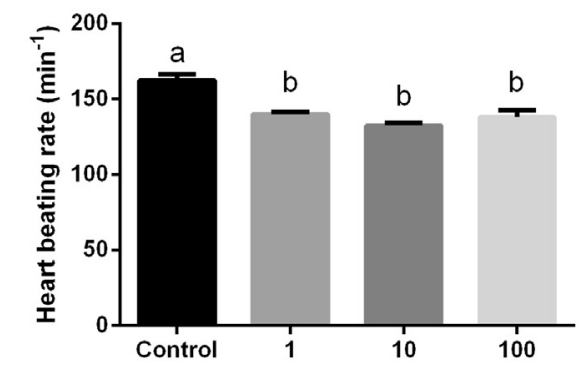

c)

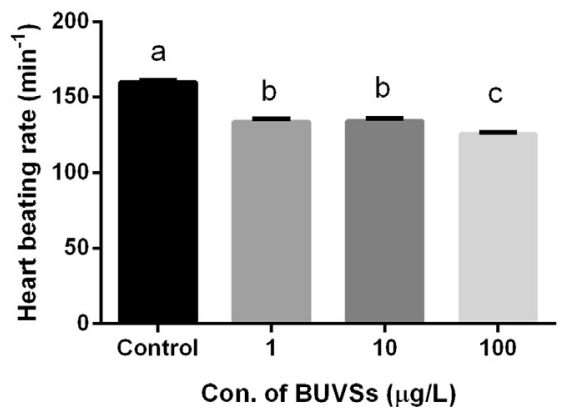

b)

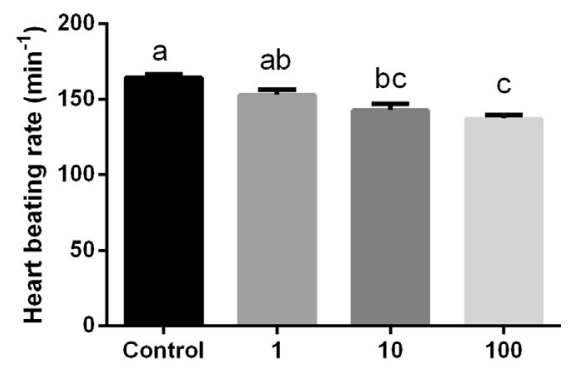

d)

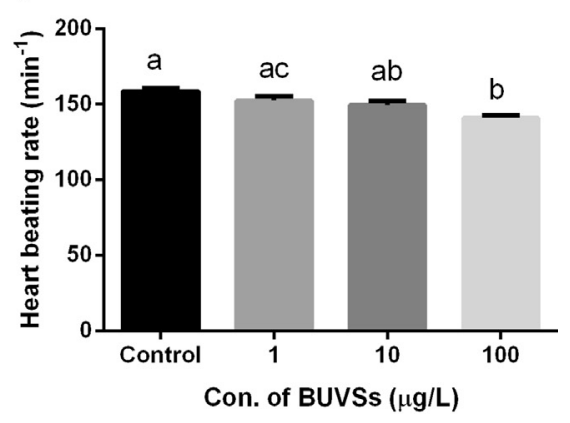

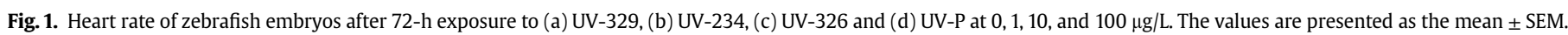
Different letters denote significant differences $(\mathrm{p}<0.05)$ from the control.

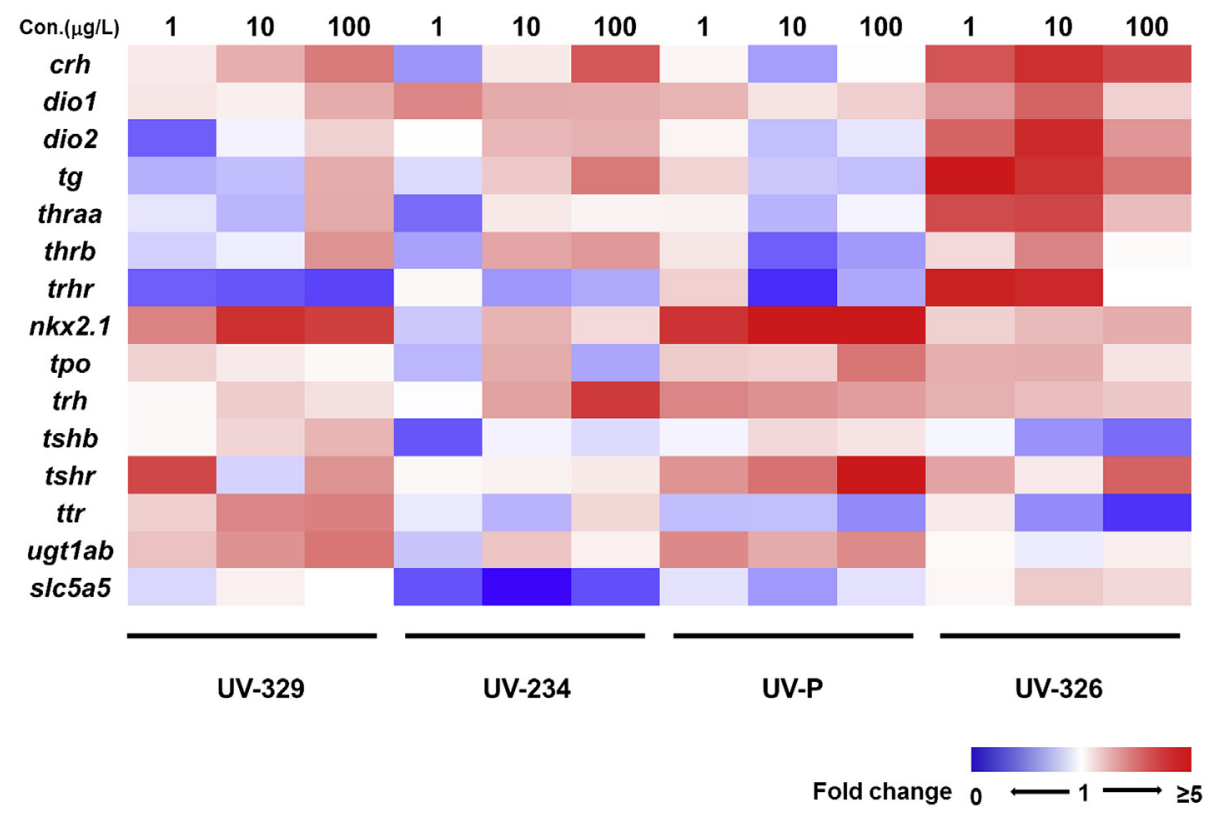

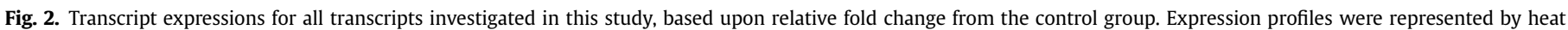

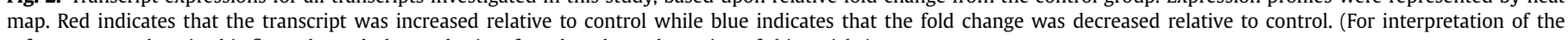
references to colour in this figure legend, the reader is referred to the web version of this article.)

organic substance, regulation of transcription from RNA polymerase II promoter, and intracellular receptor signaling pathway among others (Fig. 5b), suggesting that these processes may also be affected by UV-protecting chemicals.

\section{Discussion}

Although BUVSs have not shown ligand activity toward the human thyroid hormone receptors (Nagayoshi et al., 2015), there is evidence that these compounds may adversely affect the thyroid system of vertebrates, for example, suppression of thra in zebrafish embryos following exposure to $690 \mu \mathrm{g} / \mathrm{L}$ UV-P (Fent et al., 2014). However, a direct interaction between the thyroid receptor ligand binding domain and the environmental contaminant may not be required for thyroid system disruption. Some environmental chemicals can bind to an allosteric site on the DNA binding domain 


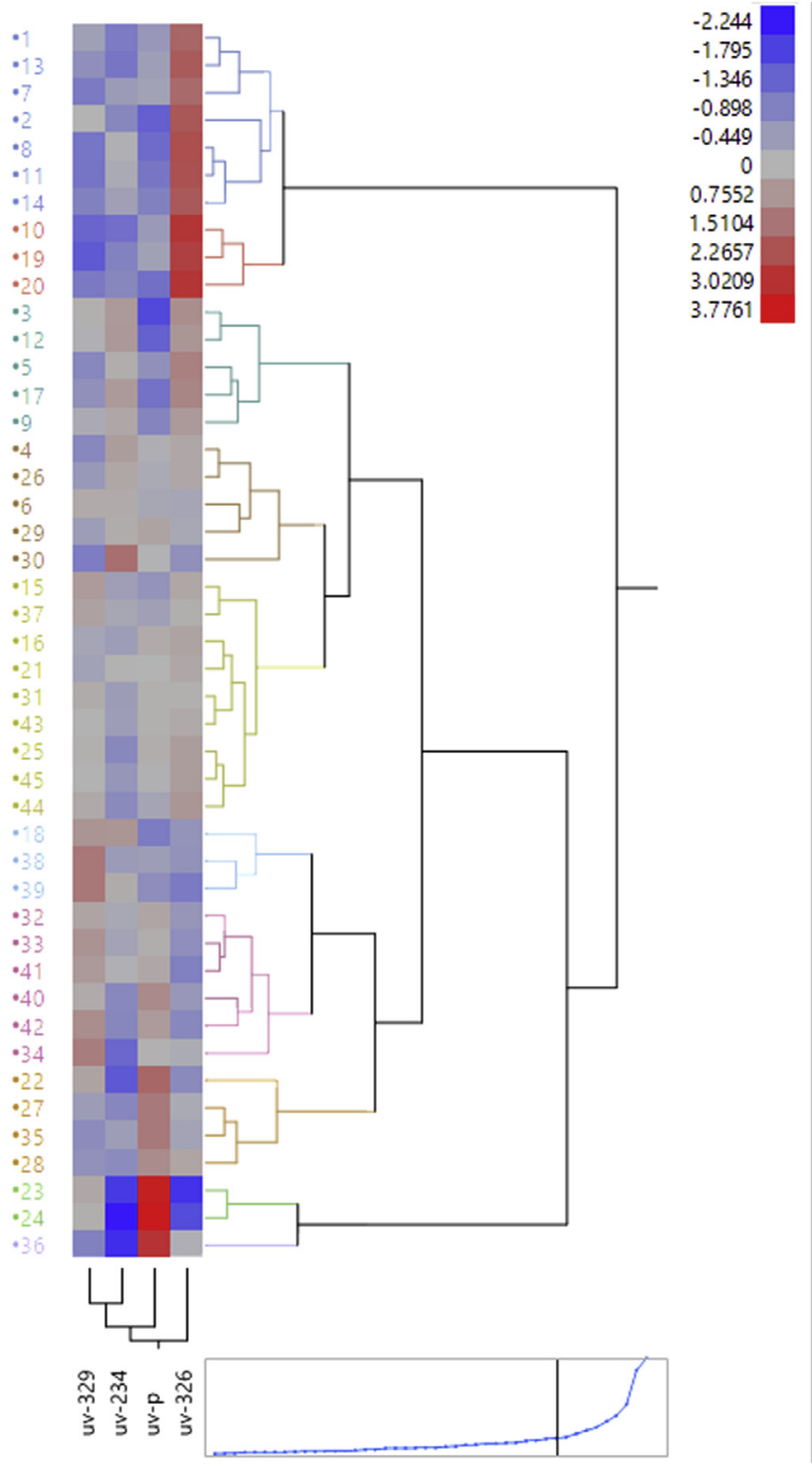

Fig. 3. Cluster analysis for UV-stabilizers, based upon relative fold change from the control group. Red indicates that the transcript was increased relative to control while blue indicates that the fold change was decreased relative to control. Transcripts in the UV-329 and the UV-324 treatment were more similar than that to UV-326 and UV-P. (For interpretation of the references to colour in this figure legend, the reader is referred to the web version of this article.)

of the thyroid hormone receptors, instead of the ligand-binding domain (Miyazaki et al., 2008). This study adds to the knowledge base of how BUVSs may interact with the thyroid system in vivo. Specifically, we examined developmental endpoints and heart rates, as well as transcriptional levels of the HPT axis-related genes in zebrafish embryos. In addition, gene networks and pathways were identified that may be associated with thyroid receptor disruption in the zebrafish, providing novel information as to the mechanisms by which BUVSs exert their effects.

There was no significant mortality nor malformations observed in zebrafish embryos during 96-h exposure to the 4 BUVSs. Conversely, the heartbeat rates were significantly decreased in animals in all treatments. In zebrafish, members of the Nk2 gene family are expressed in the developing heart (Stainier, 2001). A member of this family, $n k x 2.5$, is essential for myogenic and morphogenetic differentiation (Chen and Fishman, 1996). Although there is no evidence that $n k x 2.1$ directly regulates cardiac function, previous studies indicate that it may be involved in cardiovascular formation (Porazzi et al., 2009; Opitz et al., 2012). Early thyroid development has been found to proceed in intimate contact with cardiovascular development; thyroid budding from the pharyngeal floor is tightly coordinated with the descent of the heart (Opitz et al., 2012). In the zebrafish, $n k x 2.1$ is expressed close to the heart primordium, and cells expressing $n k \times 2.1$ comprise the region corresponding to the thyroid bud (Porazzi et al., 2009). In the current study, predicted biological process showed that regulation of cardiac chamber formation and morphogenesis, as well as cardiac muscle cell proliferation were associated with nkx2.1 (Supplemental Table S6). We hypothesize that the decreased heart rate due to BUVSs is related to the increased $n k x 2.1$ levels in this study. A previous study also demonstrated that the reduction of heart rates was accompanied by an increase in $n k x 2.1$ levels in zebrafish embryos exposed to tris(1,3-dichloro-2-propyl) phosphate (TDCPP) (Wang et al., 2013b). Furthermore, in previous studies, BUVSs show agonistic activities toward aryl hydrocarbon receptor $(A h R)$, which is the nuclear receptor mediating the toxicity of 2,3,7,8-tetrachlorodibenzo-p-dioxin (TCDD) and polycyclic aromatic hydrocarbons (PAHs) (Fent et al., 2014; Nagayoshi et al., 2015). AHR agonist can lead to cardiac dysfunction in zebrafish embryos, and cardiotoxicity is among the earliest responses to such chemicals (Antkiewicz et al., 2005; Incardona et al., 2004). These results suggest that BUVSs are likely to cause cardiac dysfunction in early stage of development in fish, and this may result in developmental toxicity after long-term exposure.

Transcriptional levels of the HPT axis-related genes were differentially regulated by UV-stabilizers, but the response of the transcripts varied depending upon the compound and concentration tested. The response pattern for genes in embryos exposed to UV-234 and UV-329 were more similar compared to UV-P and UV326 , and UV-326 was most divergent from the others in terms of expression response. Zhuang et al. (2017) recently reported on the anti-androgenic activity of 8 BUVSs (UV-P, 1HBT, UV-234, UV-326, UV-327, UV-328, UV-329 and UV-350) in the presence of human CYP3A4 enzyme; anti-androgenic activity of UV-328 is enhanced markedly after metabolism while that of UV-P is reduced. Other BUVSs tested in the study show no anti-androgenic activity, either before or after metabolism. These results suggest that minor structural differences among BUVSs may induce different toxic and endocrine activities related to gene expression.

In this study, mRNA levels of thyroid hormone receptors (thraa and thrb) in zebrafish embryos were significantly altered following UV-234, UV-326, UV-329 and UV-P treatments. In a previous study, Fent et al. (2014) reported a down-regulation of thra transcript in zebrafish eleuthero-embryos after $144 \mathrm{~h}$ exposure to $690 \mu \mathrm{g} / \mathrm{L} \mathrm{UV}-\mathrm{P}$, while no change was observed for this transcript after exposure to $84 \mu \mathrm{g} / \mathrm{L}$ UV-326. In the present study, there were no significant alterations in transcript levels of thraa detected across each of the concentrations of UV-P, while down-regulation was observed in embryos following exposure to 1 and $10 \mu \mathrm{g} / \mathrm{L}$ UV-326. It has been reported that differential transcriptional effects on the HPT axis in zebrafish embryos/larvae can be observed after chemical exposure at different stage ( $0 \mathrm{hpf}$ to $96 \mathrm{hpf}$ or $96 \mathrm{hpf}$ to $8 \mathrm{dpf}$ ) (Chan and Chan, 2012). The inconsistent results between the current study and that of Fent et al. (2014) may suggest that effects on the HPT axis by BUVSs may be time-sensitive and related to the ontogeny of the thyroid system. In addition, thrb was up-regulated in one or two doses after UV-234, UV-326 and UV-329 exposure, whereas, it was down-regulated in the $10 \mu \mathrm{g} / \mathrm{L}$ UV-P group. The embryonic to larval transitory phase in zebrafish is characterized by its dependency on 
a)

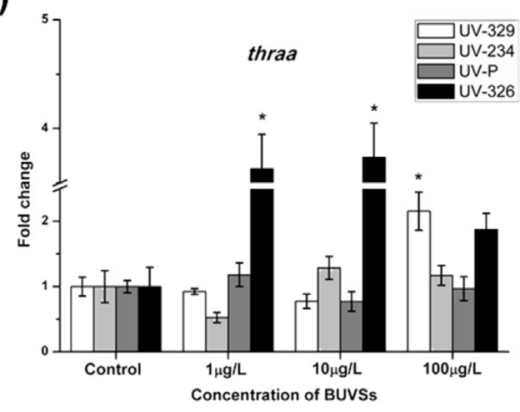

b)

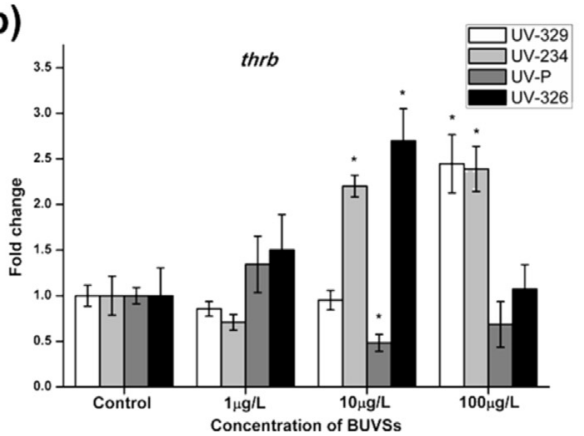

c)

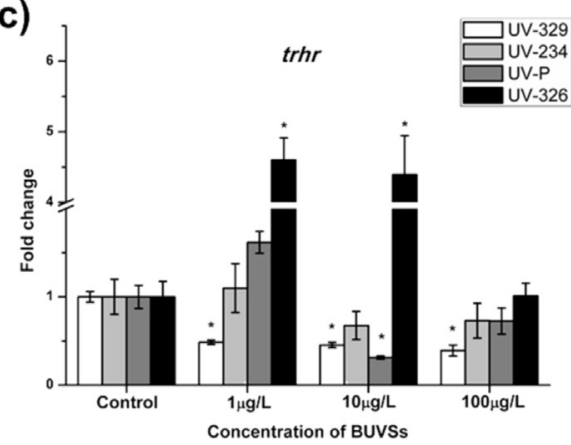

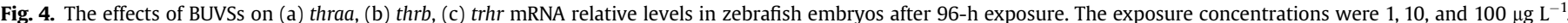

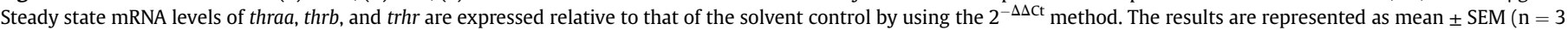
replicates).

a)

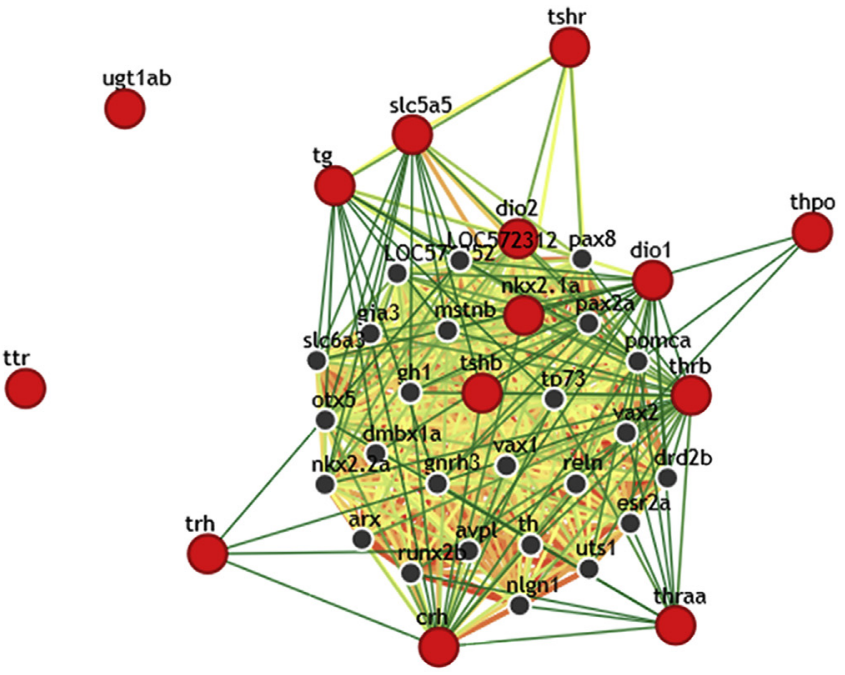

b)

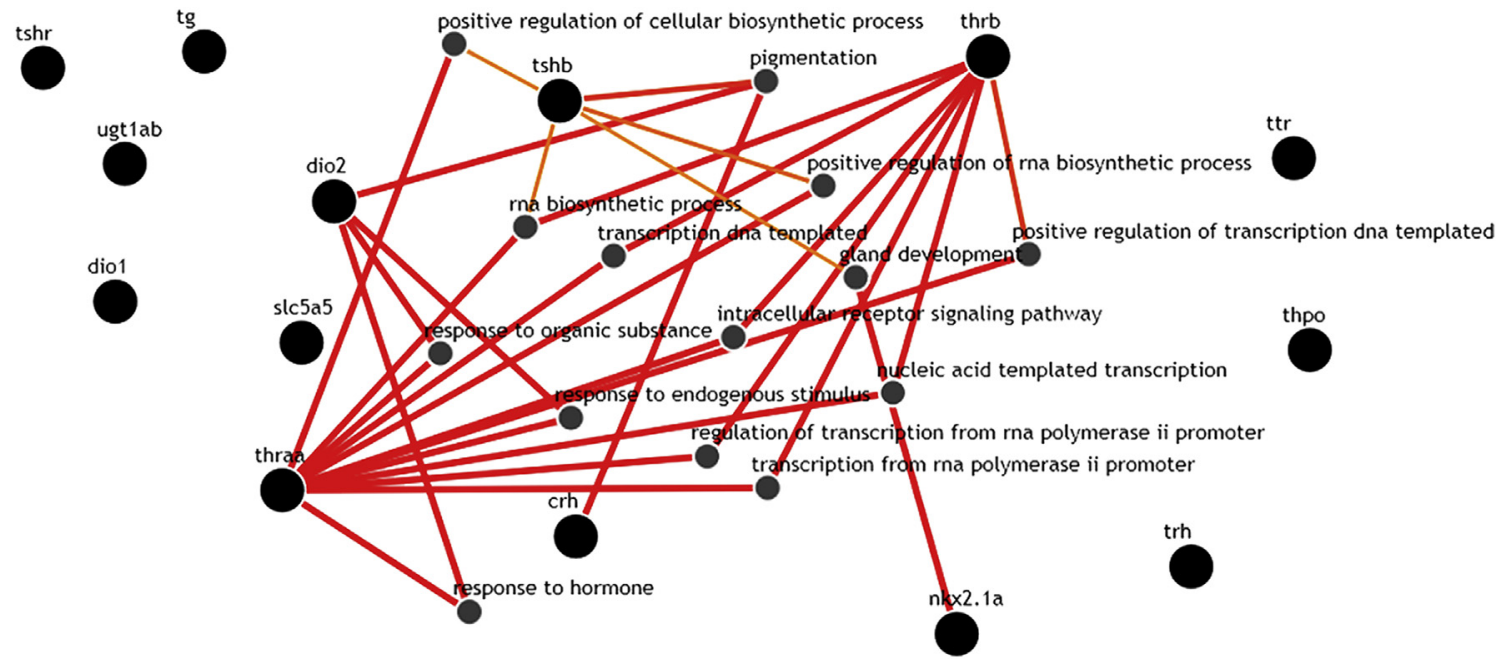

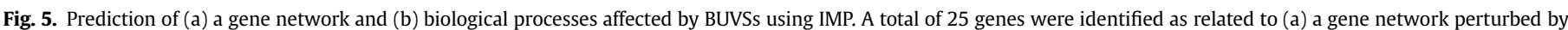

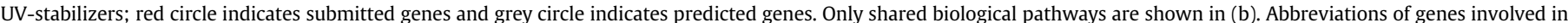
the gene network are provided in Table S3. (For interpretation of the references to colour in this figure legend, the reader is referred to the web version of this article.)

the timely synthesis of thyroid hormone and the concomitant autoregulated increase in thrb levels (Liu and Chan, 2002). Embryonic exposure to multiple chemicals (e.g. difenoconazole and 6- hydroxy-2, 2', 4, 4'-tetrabromodiphenyl ether) appears to perturb the expression of thrb and results in abnormal development in zebrafish (Liang et al., 2015; Macaulay et al., 2015). These results 
indicate that BUVSs may disturb the thyroid hormone receptor pathway and induce developmental toxicity.

Genes involved in thyroid hormone regulation (trh, trhr, tshb, and $t$ shr), synthesis (slc5a5 and $t g$ ), and metabolism (dio1 and dio2) were also examined in this study. The homeostasis of thyroid hormones is predominantly regulated by the concentration of thyroid stimulating hormone (TSH, encoded by tshb gene). The secretion of TSH is stimulated by thyrotropin-releasing hormone (encoded by trh gene) from the hypothalamus (Porazzi et al., 2009). In addition, iodothyronine deiodinases (Dio1 and Dio2) are also important regulators in homeostasis of thyroid hormones in fish, which can convert T4 to T3 in outer ring-deiodinating pathway (Orozco and Valverde, 2005). In the present study, trh, trhr, tshr, dio1 and dio2 levels were significantly affected by BUVSs. However, tshb expressions generally remained unchanged following exposure to the chemical, although there was a slight decrease of tshb in $1 \mu \mathrm{g} / \mathrm{L} \mathrm{UV}-234$ group. This corresponds to data in zebrafish embryos exposed to progesterone and norgestrel (Liang et al., 2015) in that this transcript may be relatively insensitive to hormonal regulation. We assumed that BUVSs might exert direct effects on transcriptional levels of HPT axis-related genes, instead of disrupting the expression of genes by altering TSH level. In vertebrates, expression of slc5a5 and $t g$ is regulated by $n k x 2.1$ (Zoeller et al., 2007). Many cases show that upregulated $n k \times 2.1$ level is associated with an increase of slc5a5 and $\operatorname{tg}$ (Yu et al., 2010; Wang et al., 2013b). However, in this study, there was a general up-regulation of $n k x 2.1$ and $t g$ following BUVSs exposure, while slc5a5 was down-regulated after UV-234 exposure. These data are consistent with a previous study in zebrafish larvae exposed to Bisphenol AF (Tang et al., 2015). The authors concluded that compromised iodide uptake, which was attributed to the down-regulation of slc5a5 gene, results in the accumulation of TG. Taken together, our results and that of previous studies indicate that BUVSs may have adverse effects on the homeostasis of thyroid hormones in fish. Additional physiological endpoints of BUVSs, such as thyroid hormone levels, should be evaluated in forthcoming experiments in order to better discern the effects of BUVSs on thyroid hormone signaling.

Predicted gene network construction indicated that the main biological processes/diseases that BUVSs might adversely affect included response to organic substance, regulation of transcription from RNA polymerase II promoter, intracellular receptor signaling pathway and hypothyroidism. Environmental chemicals are proposed to affect thyroid homeostasis by interfering with thyroid hormone signaling (Miller et al., 2009). These mechanisms can include alterations at the receptor level, binding to transport proteins, altering cellular uptake mechanisms or modifying the metabolism of thyroid hormones (Boas et al., 2006). Studies have demonstrated that polychlorinated biphenyls, dioxins and furans, chemicals of which there are thyroid disrupting activities, cause hypothyroidism in exposed animals (Boas et al., 2006). In this study, we detected significant changes in the expression of thyroid hormone receptors and other HPT axis-related genes. Similar to some thyroid disrupting chemicals (e.g. bisphenol A), BUVSs may exert thyroid disrupting effects by interfering with the regulation of transcription of thyroid hormone receptors, and thus adversely impact intracellular receptor signaling pathway. In addition, the significant decrease in heart rates after BUVSs exposure provides some phenotype evidence for metabolic disruption. Future investigations should be designed to better explore these disruptions more completely.

In conclusion, UV-234, UV-326, UV-329 and UV-P can significantly reduce the heart beat rates of zebrafish embryos. Moreover, the transcription levels of genes involved in the HPT axis were affected by 4 different BUVSs, each of which showed a chemicalspecific effect on gene expression. Noteworthy was that the
mRNA levels of thyroid hormone receptors in zebrafish embryos were significantly affected to some degree after exposure to one of UV-234, UV-326, UV-329 and UV-P treatments. We hypothesize that BUVSs may induce thyroid disrupting effects through toxicological pathways including response to organic substance, regulation of transcription from RNA polymerase II promoter and intracellular receptor signaling pathway, each proposed to be related to hypothyroidism. Novel genes identified in predicted gene networks such as avpl, runx $2 b$, uts $1 d r d 2 b$ and arx also provide insight into the mechanisms of thyroid disrupting toxicity of BUVSs and perhaps other environmental contaminants that affect the thyroid system.

\section{Acknowledgements}

This work was supported by the National Natural Science Foundation of China (21507064), the Natural Science Foundation of Inner Mongolia Autonomous Region of China (2015MS0202), and the University of Florida, College of Veterinary Medicine. The support provided by China Scholarship Council (CSC) during a visit of Xuefang Liang (No. 201608155003) to University of Florida is acknowledged.

\section{Appendix A. Supplementary data}

Supplementary data related to this article can be found at http:// dx.doi.org/10.1016/j.chemosphere.2017.05.015.

\section{References}

Antkiewicz, D.S., Burns, C.G., Carney, S.A., Peterson, R.E., Heideman, W., 2005. Heart malformation is an early response to TCDD in embryonic zebrafish. Toxicol. Sci. 84, 368-377.

Asimakopoulos, A.G., Bletsou, A.A., Wu, O., Thomaidis, N.S., Kannan, K., 2013a. Determination of benzotriazoles and benzothiazoles in human urine by liquid chromatography-tandem mass spectrometry. Anal. Chem. 85, 441-448.

Asimakopoulos, A.G., Wang, L., Thomaidis, N.S., Kannan, K., 2013b. Benzotriazoles and benzothiazoles in human urine from several countries: a perspective on occurrence, biotransformation, and human exposure. Environ. Int. 59, 274-281.

Blanton, M.L., Specker, J.L., 2007. The hypothalamic-pituitary-thyroid (HPT) axis in fish and its role in fish development and reproduction. Crit. Rev. Toxicol. 37, 97-115.

Boas, M., Feldt-Rasmussen, U., Skakkebæk, N.E., Main, K.M., 2006. Environmental chemicals and thyroid function. Eur. J. Endocrinol. 154, 599-611.

Cantwell, M.G., Sullivan, J.C., Katz, D.R., Burgess, R.M., Bradford Hubeny, J., King, J. 2015. Source determination of benzotriazoles in sediment cores from two urban estuaries on the Atlantic Coast of the United States. Mar. Pollut. Bull. 101, $208-218$.

Carpinteiro, I., Abuín, B., Rodríguez, I., Cela, R., Ramil, M., 2010. Headspace solidphase microextraction followed by gas chromatography tandem mass spectrometry for the sensitive determination of benzotriazole UV stabilizers in water samples. Anal. Bioanal. Chem. 397, 829-839.

Carpinteiro, I., Ramil, M., Rodríguez, I., Nogueira, J.M.F., 2012. Combining stir-bar sorptive extraction and large volume injection-gas chromatography-mass spectrometry for the determination of benzotriazole UV stabilizers in wastewater matrices. J. Sep. Sci. 35, 459-467.

Chan, W.K., Chan, K.M., 2012. Disruption of the hypothalamic-pituitary-thyroid axis in zebrafish embryo-larvae following waterborne exposure to BDE-47, TBBPA and BPA. Aquat. Toxicol. 108, 106-111.

Chen, J.N., Fishman, M.C., 1996. Zebrafish tinman homolog demarcates the heart field and initiates myocardial differentiation. Development 122, 3809-3816.

Fent, K., Chew, G., Li, J., Gomez, E., 2014. Benzotriazole UV-stabilizers and benzotriazole: antiandrogenic activity in vitro and activation of aryl hydrocarbon receptor pathway in zebrafish eleuthero-embryos. Sci. Total Environ. 482-483, 125-136.

García-Guerra, R.B., Montesdeoca-Esponda, S., Sosa-Ferrera, Z., Kabir, A., Furton, K.G., Santana-Rodríguez, J.J., 2016. Rapid monitoring of residual UVstabilizers in seawater samples from beaches using fabric phase sorptive extraction and UHPLC-MS/MS. Chemosphere 164, 201-207.

Incardona, J.P., Collier, T.K., Scholz, N.L., 2004. Defects in cardiac function precede morphological abnormalities in fish embryos exposed to polycyclic aromatic hydrocarbons. Toxicol. Appl. Pharmacol. 196, 191-205.

Kameda, Y., Kimura, K., Miyazaki, M., 2011. Occurrence and profiles of organic sunblocking agents in surface waters and sediments in Japanese rivers and lakes. Environ. Pollut. 159, 1570-1576. 
Kawamura, Y., Ogawa, Y., Nishimura, T., Kikuchi, Y., Nishikawa, J., Nishihara, T., Tanamoto, K., 2003. Estrogenic activities of UV stabilizers used in food contact plastics and benzophenone derivatives tested by the yeast two-hybrid assay. J. Health Sci. 49, 205-212.

Kim, J.W., Chang, K.H., Isobe, T., Tanabe, S., 2011a. Acute toxicity of benzotriazole ultraviolet stabilizers on freshwater crustacean (Daphnia pulex). J. Toxicol. Sci. 36, 247-251.

Kim, J.W., Isobe, T., Ramaswamy, B.R., Chang, K.H., Amano, A., Miller, T.M., Siringan, F.P., Tanabe, S., 2011b. Contamination and bioaccumulation of benzotriazole ultraviolet stabilizers in fish from Manila Bay, the Philippines using an ultra-fast liquid chromatography-tandem mass spectrometry. Chemosphere 85, 751-758.

Kim, J.W., Ramaswamy, B.R., Chang, K.H., Isobe, T., Tanabe, S., 2011c. Multiresidue analytical method for the determination of antimicrobials, preservatives, benzotriazole UV stabilizers, flame retardants and plasticizers in fish using ultra high performance liquid chromatography coupled with tandem mass spectrometry. J. Chromatogr. A 1218, 3511-3520.

Kimmel, C.B., Ballard, W.W., Kimmel, S.R., Ullmann, B., Schilling, T.F., 1995. Stages of embryonic development of the zebrafish. Dev. Dyn. 203, 253-310.

Lai, H.J., Ying, G.G., Ma, Y.B., Chen, Z.F., Chen, F., Liu, Y.S., 2014. Occurrence and dissipation of benzotriazoles and benzotriazole ultraviolet stabilizers in biosolid-amended soils. Environ. Toxicol. Chem. 33, 761-767.

Langford, K.H., Reid, M.J., Fjeld, E., Øxnevad, S., Thomas, K.V., 2015. Environmental occurrence and risk of organic UV filters and stabilizers in multiple matrices in Norway. Environ. Int. 80, 1-7.

Lee, S., Kim, S., Park, J., Kim, H.J., Jae Lee, J., Choi, G., Choi, S., Kim, S., Young Kim, S., Choi, K., Kim, S., Moon, H.B., 2015. Synthetic musk compounds and benzotriazole ultraviolet stabilizers in breast milk: occurrence, time-course variation and infant health risk. Environ. Res. 140, 466-473.

Liang, X., Yu, L., Gui, W., Zhu, G., 2015. Exposure to difenoconazole causes changes of thyroid hormone and gene expression levels in zebrafish larvae. Environ. Toxicol. Pharmacol. 40, 983-987.

Liu, Y.S., Ying, G.G., Shareef, A., Kookana, R.S., 2011. Simultaneous determination of benzotriazoles and ultraviolet filters in ground water, effluent and biosolid samples using gas chromatography-tandem mass spectrometry. J. Chromatogr. A $1218,5328-5335$.

Liu, Y.W., Chan, W.K., 2002. Thyroid hormones are important for embryonic to larval transitory phase in zebrafish. Differentiation 70, 36-45.

Livak, K.J., Schmittgen, T.D., 2001. Analysis of relative gene expression data using real-time quantitative PCR and the $2^{-\triangle \Delta C T}$ method. Methods 25, 402-408.

Lu, Z., Peart, T.E., Cook, C.J., De Silva, A.O., 2016. Simultaneous determination of substituted diphenylamine antioxidants and benzotriazole ultra violet stabilizers in blood plasma and fish homogenates by ultra high performance liquid chromatography-electrospray tandem mass spectrometry. J. Chromatogr. A 1461, 51-58.

Macaulay, L.J., Chen, A., Rock, K.D., Dishaw, L.V., Dong, W., Hinton, D.E., Stapleton, H.M., 2015. Developmental toxicity of the PBDE metabolite 6-OHBDE-47 in zebrafish and the potential role of thyroid receptor $\beta$. Aquat. Toxicol. 168, 38-47.

Miller, M.D., Crofton, K.M., Rice, D.C., Zoeller, R.T., 2009. Thyroid-disrupting chemicals: interpreting upstream biomarkers of adverse outcomes. Environ. Health Perspect. 117, 1033.

Miyazaki, W., Iwasaki, T., Takeshita, A., Tohyama, C., Koibuchi, N., 2008. Identification of the functional domain of thyroid hormone receptor responsible for polychlorinated biphenyl-mediated suppression of its action in vitro. Environ. Health Perspect. 116, 1231-1236.

Montesdeoca-Esponda, S., Sosa-Ferrera, Z., Santana-Rodríguez, J.J., 2012. On-line solid-phase extraction coupled to ultra-performance liquid chromatography with tandem mass spectrometry detection for the determination of benzotriazole UV stabilizers in coastal marine and wastewater samples. Anal. Bioanal. Chem. 403, 867-876.

Morohoshi, K., Yamamoto, H., Kamata, R., Shiraishi, F., Koda, T., Morita, M., 2005. Estrogenic activity of 37 components of commercial sunscreen lotions evaluated by in vitro assays. Toxicol. In Vitro 19, 457-469.

Nagayoshi, H., Kakimoto, K., Takagi, S., Konishi, Y., Kajimura, K., Matsuda, T., 2015. Benzotriazole ultraviolet stabilizers show potent activities as human aryl hydrocarbon receptor ligands. Environ. Sci. Technol. 49, 578-587.

Nakata, H., Murata, S., Filatreau, J., 2009. Occurrence and concentrations of benzotriazole UV stabilizers in marine organisms and sediments from the Ariake
Sea, Japan. Environ. Sci. Technol. 43, 6920-6926.

Nakata, H., Shinohara, R., Murata, S., Watanabe, M., 2010. Detection of benzotriazole UV stabilizers in the blubber of marine mammals by gas chromatography-high resolution mass spectrometry (GC-HRMS). J. Environ. Monit. 12, 2088-2092.

Nakata, H., Shinohara, R., Nakazawa, Y., Isobe, T., Sudaryanto, A., Subramanian, A., Tanabe, S., Zakaria, M.P., Zheng, G.J., Lam, P.K., 2012. Asia-Pacific mussel watch for emerging pollutants: distribution of synthetic musks and benzotriazole UV stabilizers in Asian and US coastal waters. Mar. Pollut. Bull. 64, 2211-2218.

Nichols, J.W., Breen, M., Denver, R.J., DiStefano, J.J., Edwards, J.S., Hoke, R.A., Volz, D.C., Zhang, X., 2011. Predicting chemical impacts on vertebrate endocrine systems. Environ. Toxicol. Chem. 30, 39-51.

Opitz, R., Maquet, E., Huisken, J., Antonica, F., Trubiroha, A., Pottier, G., Janssens, V., Costagliola, S., 2012. Transgenic zebrafish illuminate the dynamics of thyroid morphogenesis and its relationship to cardiovascular development. Dev. Biol. 372, 203-216.

Orozco, A., Valverde, R.C., 2005. Thyroid hormone deiodination in fish. Thyroid 15, 799-813.

Porazzi, P., Calebiro, D., Benato, F., Tiso, N., Persani, L., 2009. Thyroid gland development and function in the zebrafish model. Mol. Cell Endocrinol. 312, 14-23.

Power, D.M., Llewellyn, L., Faustino, M., Nowell, M.A., Bjornsson, B.T. Einarsdottir, I.E., Canario, A.V., Sweeney, G.E., 2001. Thyroid hormones in growth and development of fish. Comp. Biochem. Physiol. C Toxicol. Pharmacol. 130, 447-459.

Raldúa, D., Thienpont, B., Babin, P.J., 2012. Zebrafish eleutheroembryos as an alternative system for screening chemicals disrupting the mammalian thyroid gland morphogenesis and function. Reprod. Toxicol. 33, 188-197.

Ramos, S., Homem, V., Alves, A., Santos, L., 2016. A review of organic UV-filters in wastewater treatment plants. Environ. Int. 86, 24-44.

Reimers, M.J., Flockton, A.R., Tanguay, R.L., 2004. Ethanol- and acetaldehydemediated developmental toxicity in zebrafish. Neurotoxicol. Teratol. 26, $769-781$.

Ruan, T., Liu, R., Fu, Q., Wang, T., Wang, Y., Song, S., Wang, P., Teng, M., Jiang, G., 2012. Concentrations and composition profiles of benzotriazole UV stabilizers in municipal sewage sludge in China. Environ. Sci. Technol. 46, 2071-2079.

Song, S., Ruan, T., Wang, T., Liu, R., Jiang, G., 2014. Occurrence and removal of benzotriazole ultraviolet stabilizers in a wastewater treatment plant in China. Environ. Sci. Process Impacts 16, 1076-1082.

Stainier, D.Y.R., 2001. Zebrafish genetics and vertebrate heart formation. Nat. Rev. Genet. 2, 39-48.

Tang, T., Yang, Y., Chen, Y., Tang, W., Wang, F., Diao, X., 2015. Thyroid disruption in zebrafish larvae by short-term exposure to bisphenol AF. Int. J. Environ. Res. Public Health 12, 13069-13084.

Wang, L., Asimakopoulos, A.G., Kannan, K., 2015. Accumulation of 19 environmental phenolic and xenobiotic heterocyclic aromatic compounds in human adipose tissue. Environ. Int. 78, 45-50.

Wang, L., Asimakopoulos, A.G., Moon, H.B., Nakata, H., Kannan, K., 2013a. Benzotriazole, benzothiazole, and benzophenone compounds in indoor dust from the United States and East Asian countries. Environ. Sci. Technol. 47, 4752-4759.

Wang, Q., Liang, K., Liu, J., Yang, L., Guo, Y., Liu, C., Zhou, B., 2013b. Exposure of zebrafish embryos/larvae to TDCPP alters concentrations of thyroid hormones and transcriptions of genes involved in the hypothalamic-pituitary-thyroid axis. Aquat. Toxicol. 126, 207-213.

Wick, A., Jacobs, B., Kunkel, U., Heininger, P., Ternes, T.A., 2016. Benzotriazole UV stabilizers in sediments, suspended particulate matter and fish of German rivers: new insights into occurrence, time trends and persistency. Environ. Pollut. 212, 401-412.

Yu, L., Deng, J., Shi, X., Liu, C., Yu, K., Zhou, B., 2010. Exposure to DE-71 alters thyroid hormone levels and gene transcription in the hypothalamic-pituitary-thyroid axis of zebrafish larvae. Aquat. Toxicol. 97, 226-233.

Zhang, Z., Ren, N., Li, Y.F., Kunisue, T., Gao, D., Kannan, K., 2011. Determination of benzotriazole and benzophenone UV filters in sediment and sewage sludge. Environ. Sci. Technol. 45, 3909-3916.

Zhuang, S., Lv, X., Pan, L., Lu, L., Ge, Z., Wang, J., Wang, J., Liu, J., Liu, W., Zhang, C., 2017. Benzotriazole UV 328 and UV-P showed distinct antiandrogenic activity upon human CYP3A4-mediated biotransformation. Environ. Pollut. 220 (Part A), 616-624.

Zoeller, R.T., Tan, S.W., Tyl, R.W., 2007. General background on the hypothalamicpituitary-thyroid (HPT) axis. Crit. Rev. Toxicol. 37, 11-53. 